\title{
Design and fabrication of a high-density 2D fiber array for holographic switching applications
}

\author{
Cedric Letort \\ Bruno Vinouze \\ Bruno Fracasso \\ ENST Bretagne \\ Optics Department \\ UMR 6082 Foton \\ CS 83818-29238, Brest Cedex 3, France \\ E-mail: cedric.letort@enst-bretagne.fr
}

\begin{abstract}
Holographic two-dimensional beam steering using a pixellated spatial light modulator (SLM) is a flexible and accurate technique to implement advanced space-switching, building-block functions for optical network nodes. We present the design of a specific fiber array for largecapacity holographic switches. We use two-dimensional and irregular array topology to optimize the space-bandwidth product of the SLM and to avoid crosstalk due to the coupling of higher diffraction orders. The fiber-array module is integrated by inserting the fibres through a pair of etched silicon plates aligned and fixed using a steel mount. The device is tested through near-field and fiber-to-fiber free space coupling tests. An average fiber-to-fiber insertion loss of $1 \mathrm{~dB}$ is achieved for a prototype containing 64 fibers. ( 2008 Society of Photo-Optical Instrumentation Engineers. [DOI: 10.1117/1.2911718]
\end{abstract}

Subject terms: optical fiber array; wavelength-selective switch; spatial light modulator; liquid crystal spatial; light modulator; binary-phase hologram.

Paper 070648R received Aug. 1, 2007; revised manuscript received Nov. 21, 2007; accepted for publication Jan. 2, 2008; published online Apr. 30, 2008.

\section{Introduction}

In the last few years, dense wavelength-division multiplexed (DWDM)-based optical telecommunications networks have been massively deployed to face the dramatic traffic rise generated by Internet services. The introduction of transparent cross-connecting nodes in the optical network is increasingly put forward to obtain more flexibility and reconfigurability at the optical layer. ${ }^{1} \mathrm{~A}$ key issue then is the availability of flexible all-optical switching modules, in which different operations are implemented, such as space routing, wavelength extraction, or power equalization.

In this context, the wavelength-selective switch (WSS) appears as a key technology that performs both selective routing and dynamic power control of the optical channels. ${ }^{2}$ Although planar ligthwave architectures ${ }^{3}$ yield WSS implementations with high density integration, free space-based solutions are currently the most promising in terms of insertion loss $(\approx 5 \mathrm{~dB})$, channel isolation $(40 \mathrm{~dB})$, and polarization sensitivity (PDL $\leq 1 \mathrm{~dB}$ ). Technologically speaking, the dynamic spatial-routing function of the optical channels is performed through beam deflection using either microelectromechanical systems (MEMS) mirror arrays ${ }^{4}$ or a two-dimensional liquid crystal spatial light modulator (LC-SLM). ${ }^{5}$

Within that frame and before the advent of WSS, holographic beam steering using LC-SLMs had been demonstrated as an interesting technique to increase the accuracy, repeatability, and stability of micro-beam steerers within optical space switches. ${ }^{6,7}$ Optical space switching was also backed up with dynamic wavelength-filtering capabilities. This was demonstrated for a one-dimensional $1 \times 14$ holographic switch exhibiting $8.5-\mathrm{dB}$ loss with multicast capabilities. ${ }^{7}$ The major limitation was a rather poor channel isolation level $(-21 \mathrm{~dB}$ max), due to a one-dimensional beam steering and fiber array geometry. Moving to a twodimensional geometry was then considered a decisive option to increase the switch capacity while decreasing the cross-talk levels. The first step has already been performed with the specific adaptation of a two-dimensional LC-SLM for telecommunications uses on a pre-industrial levels. ${ }^{8}$ The device was a silicon backplane $1280 \times 1024$ pixel $($ SXGA) ferroelectric spatial light modulator operating in the $1.55-\mu \mathrm{m}$ wavelength range. The second step consists of designing and fabricating a 2D fiber-array structure providing high-capacity and low-crosstalk values in the present holographic beam-steering configuration. This will be presented throughout this paper, which is organized as follows: The diffraction model for holographic beam steering is presented in Section 2 to describe the diffraction orders distribution impinging onto the output plane (replay field). This determines the constraints for the fiber-array design algorithm. Section 3 presents the design and fabrication of a specific $2 \mathrm{D}$ fiber-array prototype meeting the capacity and crosstalk requirements. The component is then tested and the experimental results are presented in Section 4.

\section{Fiber-Array Custom Design}

\subsection{Holographic Switching Setup}

The principle of a $1 \times N$ holographic optical space switch ${ }^{6,7}$ is depicted in Fig. 1. The input fiber mode is imaged onto the targeted output fiber core using a folded telecentric optical setup ( $4 f$ architecture). The positive lens is an achromat doublet coated with a $1.5-\mu \mathrm{m}$ antireflection layer, whose focal length and numerical aperture are determined to minimize aberration and vignetting of the expanded fiber-mode field. The intermediate beam deflection is performed by a reflective LC-SLM displaying phase gratings 


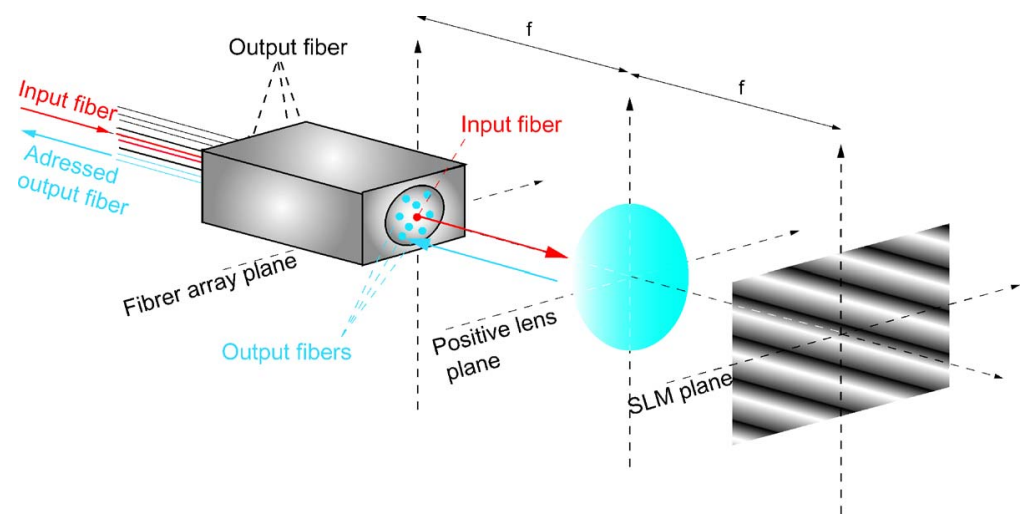

Fig. 1 Reflective holographic space-switch architecture.

(or holograms), which, since the input and output fibers are located in the same fiber array holder, permits us to limit the switch length while improving the opto-mechanical alignment procedure. The angular deviation resulting from the beam passing through the hologram is transformed into a spatial offset at the output (replay) plane. Overall, this system can be considered a fiber spatial correlator. Compared to MEMS reflective techniques, diffractive beamsteering provides motionless and high-capacity space switching with wavelength-band selection capabilities. ${ }^{7}$ Both techniques have their own advantages: Micro-mirror arrays have low loss and are intrinsically independent of the polarization and wavelength of the input light. On the other hand, LC holographic modules operate with no moving parts and can be used not only to steer optical beams but also to address several output fibers simultaneously, providing natural channel-bridging capabilities, as well as to shift the spectral response of the selected optical output. ${ }^{7}$ These two points represent a decisive advantage over MEMS technology in terms of system flexibility.

Using liquid crystal technology, various phase modulations can be achieved depending on the LC material choice. An almost continuous-phase profile can be obtained with a nematic LC, but the phase modulation depth is polarization-dependent. In contrast, a Smectic $\mathrm{C}$ liquid crystal-often referred to as ferroelectric liquid crystal (FLC) — makes the device purely polarization-insensitive, with a switching time of a few hundreds of microseconds, ${ }^{6-8}$ but to the detriment of diffraction efficiency. The recent introduction of 2D-LCOS (Liquid Crystal on Silicon) SLM devices operating in the $1.55-\mu \mathrm{m}$ telecommunication window for both $\mathrm{WSSs}^{5}$ and holographic switches ${ }^{8}$ makes the design of large-capacity (i.e., output port count $>100$ ) devices possible.

In that framework, a key step is the design of an optimized 2D single-mode fiber array that takes advantage of the high-output port count addressing capabilities of holographic deflectors.

\subsection{Holographic Beam-Steering Model}

A detailed analysis of the optical reconstruction generated by pixellated and phase-quantized LC-SLMSs can be found in the literature. ${ }^{9}$ We shall briefly present the reconstruction model for binary and pixellated hologram patterns with the purpose of determining the relevant steering parameters,

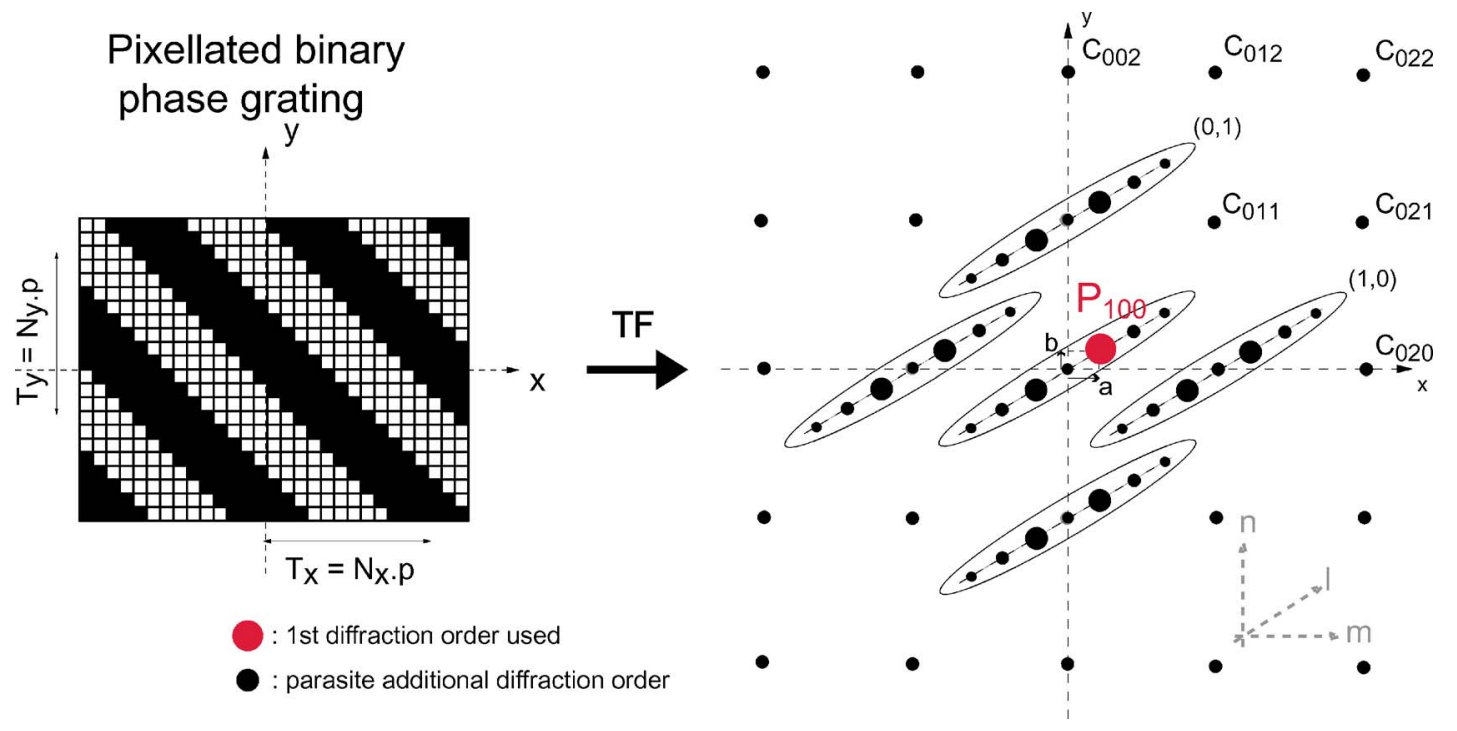

Fig. 2 Three-dimensional higher diffraction order distribution generated by a binary-phase 2D grating. 


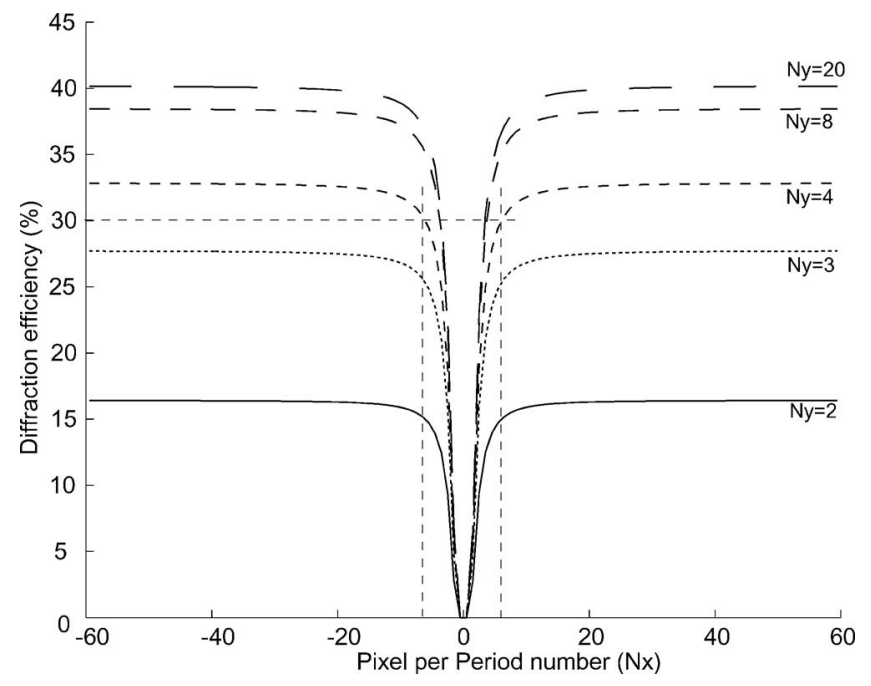

Fig. 3 Diffraction efficiency evolution versus pixel per period number $N_{x}$ as a function of $N_{y}$. Negative values correspond to symmetric gratings with respect to the $y$-axis.

namely the intensity and location of the higher diffraction orders. Using this information, we will be able to derive the beam-coupling ratio at any position of the output fiber plane and hence design a 2D fiber-array topology that minimizes the holographic switch crosstalk. Geometrical constraints like aberration and vignetting at the Fourier lens will have a limited influence if the optical setup is properly designed. $^{7}$ These parameters will hence not be considered in the model. The ideal routing holographic pattern is the blazed grating hologram that produces a unique replay of the input fiber mode at a given output fiber location. More precisely, if $\left(a_{j}, b_{j}\right)$ are the coordinates of the $j$ th output fiber (i.e., the core center location), the two-dimensional information to be displayed at the SLM plane is the periodic phase-only distribution given by

$H(x, y)=\exp \left\{-\frac{2 i \pi}{\lambda f}\left(a_{j} x+b_{j} y\right)\right\}$,

where $\lambda$ and $f$ denote, respectively, the wavelength and the focal length. The spatial periods in the $x$ and $y$ dimensions are given by $T_{x}^{j}=\lambda f / a_{j}$ and $T_{y}^{i}=\lambda f / b_{j}$. The encoding of distribution (1) onto a pixellated binary-phase function is required to physically display the steering information onto an FLC-SLM. This operation can be represented by a procedure involving three steps. The phase of the steering

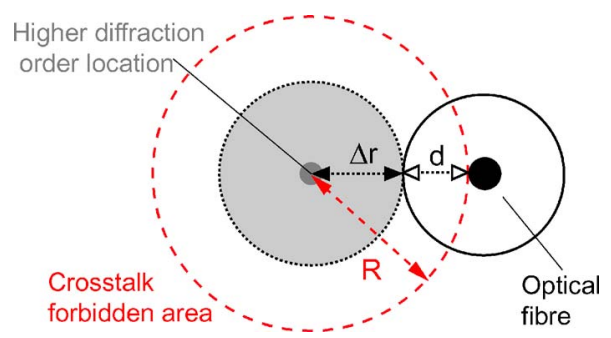

Fig. 4 Exclusion disk method to remove potential crosstalk positions from the fiber location search space. function $H$ is first quantized by comparing the real part $\mathfrak{R}(H)$ of the complex function to a threshold level $\tau$. The resulting binary phase grating $H^{q}$ takes the $\phi$ phase level if $\Re(H)<\tau$ and the $\phi+\pi$ value otherwise. The $\tau$ value controls the compression ratio $\alpha$ of the binary grating $H^{q}$. The optimized case is $\tau=0$ as it yields a balanced grating with $\alpha=0.5$, and hence no energy in the zeroth diffraction order. Then, the pixellation and dead-space (interpixel) effects are introduced. If we consider a pixel pitch value of $p$ and a pixel size of $p_{0}\left(p_{0}<p\right.$ is the size of the modulating pixel zone), the holographic steering distribution $H^{s}(x, y)$ will result from a multiplication of $H^{q}$ with a 2 Dirac distribution $\amalg_{p}(x, y)$ ( $p$ is the pixel grid/pitch), followed by the convolution of the result with a square distribution $\Pi_{p_{0}}$ of size $p_{0} \times p_{0}$, representing the pixel shape. The SLM fill factor is given by the ratio $F=\left(p_{0} / p\right)^{2}$. This finally gives us

$H^{s}(x, y)=\left\{H^{q}(x, y) \amalg_{p}(x, y)\right\} * \prod_{p_{0}}(x, y)$,

where $\Pi_{p_{0}}(x, y)=1$ if $|x| \leq p_{0}$ and $|y| \leq p_{0}$, and $\Pi_{p_{0}}(x, y)$ $=0$ elsewhere, $*$ standing for the convolution operation.

The SLM limited size will be discussed later. Note from relation (2) that the grating periods $T_{x}$ and $T_{y}$ are now quantized by the pixel pitch $p$ in the form $T_{x}=p N_{x}$ and $T_{y}$ $=p N_{y}$, with $N_{x}$ and $N_{y}$ the number of pixels per period in each dimension. The relative complex amplitudes $C_{l m n}$ and positions $P_{l m n}$ of all the replay orders can be determined by calculating the Fourier transform $\tilde{H}^{s}(\mu, \nu)$ of $H^{s}(x, y)$ from (2), yielding, ${ }^{10}$ for $l \neq 0$,

$$
\begin{aligned}
C_{l m n}= & \kappa \operatorname{sinc}(\alpha l) \operatorname{sinc}\left\{\sqrt{F}\left[m+\frac{1}{N_{x}}\right]\right\} \\
& \operatorname{sinc}\left\{\sqrt{F}\left[n+\frac{1}{N_{y}}\right]\right\} \text { and } P_{l m n}=\left(\sigma\left[m+\frac{1}{N_{x}}\right],\right. \\
& \left.\sigma\left[n+\frac{1}{N_{y}}\right]\right),
\end{aligned}
$$

where $\operatorname{sinc}(x)=\sin (\pi x) /(\pi x), \kappa=2 \iota \alpha F$, and $\sigma=\lambda f / p$.

As illustrated in Fig. 2, relation (3) represents a threedimensional diffraction-order $(l)$ distribution, where the replication is caused by the phase-level binarization, while the spatial quantization operation (pixellation) produces the $2-\mathrm{D}$ replication along with the $m$ and $n$ directions. Due to the spatial convolution generated by the $4 f$ optical setup, the input fiber mode will be spatially convolved with the $\tilde{H}^{s}(\mu, \nu)$ distribution, yielding a constellation of weighted and shifted replications as given by relation (3). The ( $l$ $=1, m=0, n=0)$ term is the main "peak" addressing the output fiber at position $(a, b)$. We can then derive from (3) the diffraction efficiency $\eta$, as a function of the grating periods $\left(N_{x}, N_{y}\right)$ :

$\eta\left(N_{x}, N_{y}\right)=\left|C_{100}\right|^{2}=\frac{4 F^{2}}{\pi^{2}} \operatorname{sinc}^{2}\left(\frac{\sqrt{F}}{N_{x}}\right) \operatorname{sinc}^{2}\left(\frac{\sqrt{F}}{N_{y}}\right)$,

where we have considered a balanced grating $(\alpha=0.5)$ for simplicity. The upper limit in $\eta$ is obtained when $N_{x}, N_{y}$ 


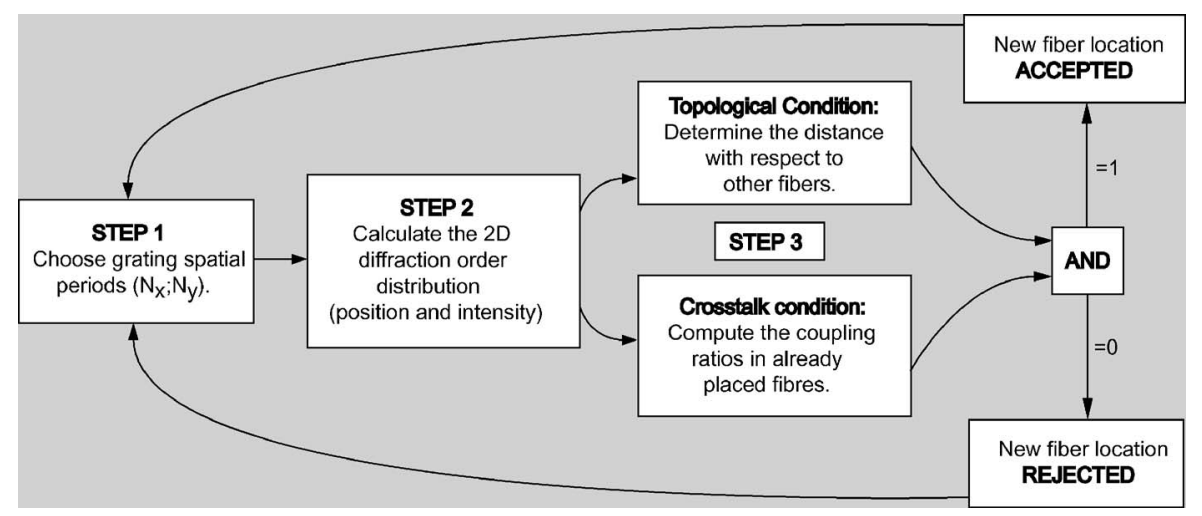

Fig. 5 Fiber core placement algorithm principle.

$\rightarrow \infty$ and $F \rightarrow 1$, yielding $\eta_{\max }=4 / \pi^{2}=0.41$. Figure 3 shows that diffraction efficiency (or steering efficiency) is relatively constant for grating period values $N_{x} \geq 4$ and decrease significantly for smaller pixel per period number. Expression (4) being symmetric with respect to $N_{y}$, we shall restrict the grating period search space to $N_{x} \geq 4$ and $N_{y} \geq 4$, which will ensure that $\eta\left(N_{x}, N_{y}\right) \geq 0.3$.

The steering model could be further refined by considering deviations in the FLC cell parameters with respect to their optimal value, e.g., cell thickness, birefringence, and tilt angle. However, such variations will not affect the crosstalk evaluation and hence the basic steering model will be kept for the remainder of the discussion. Conversely, the limited size of the SLM will modify the output plane irradiance distribution, and the correction will be included in the fiber-array design algorithm presented in the next sec- tion. The final step of the modeling will consist of calculating the coupling ratios between the input replicated modes and the output fibers. This point will be addressed in the next section.

\subsection{Crosstalk Model}

The input fiber mode replica as given by (3) will potentially generate crosstalk at the output plane. The description of the crosstalk events strongly depends on the output fiber placement process, which will be detailed in the next section. For the moment, we suppose that $N_{f}$ fibers have been placed, each corresponding to a position $\left(a_{j}, b_{j}\right)$ with 1 $\leq j \leq N_{f}$, with an addressing grating described by an $\left(N_{x}^{j}, N_{y}^{j}\right)$ pair. The question then is to know if a new grating with $\left(N_{x}^{j+1}, N_{y}^{j+1}\right)$ pixels per period is acceptable taking into

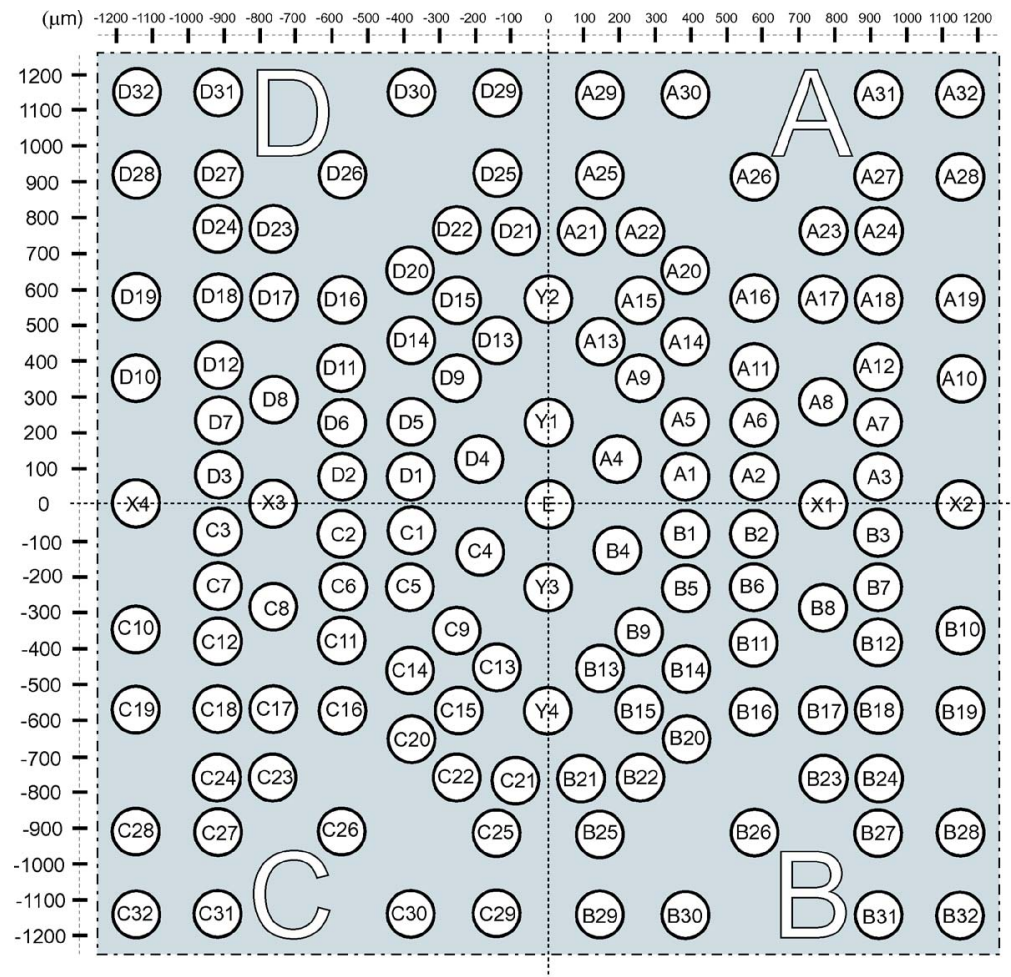

Fig. 6 Fiber array layout with 141 SM fibers placed within a $1.2 \times 1.2-\mathrm{mm}$ square. 


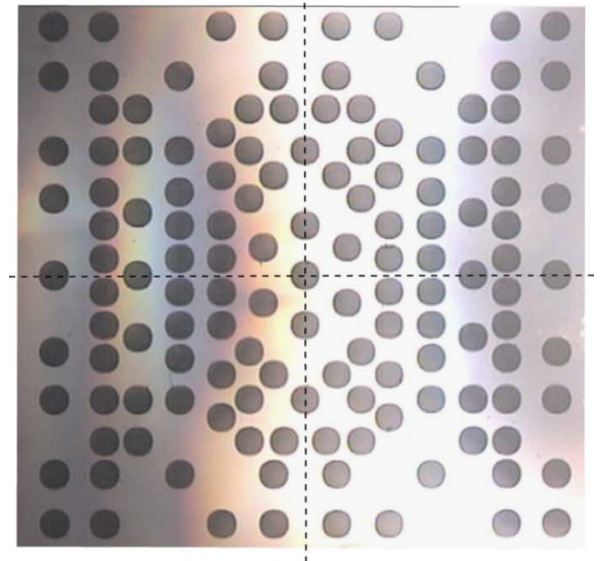

(a)

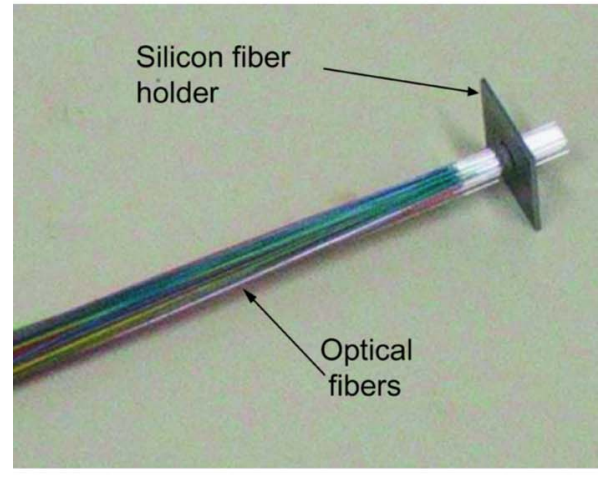

(b)

Fig. 7 (a) Top view of the bare silicon fiber holder with etched holes. (b) Side view after the fiber threading step.

account the fiber positions already set. Using its main $(1,0,0)$ th order, this grating will address a fiber position located at the output coordinates $a_{j+1}=\sigma / p N_{x}^{j+1}$ and $b_{j+1}$ $=\sigma / N_{y}^{j+1}$. Hence, two types of crosstalk conditions can be considered. First, the crosstalk between the higher orders corresponding to previously placed fibers and the virtually present tested fiber (referred to as past or P-crosstalk), and, second, the crosstalk between higher orders of the present $\left(N_{x}^{j+1}, N_{y}^{j+1}\right)$ grating and the set of previously placed $N_{f}$ fibers (referred to as future or F-crosstalk).

Crosstalk evaluation is based on the calculation of the optical intensity coupling of the incident Gaussian beams in the closest output fibers. We shall suppose that the optical setup is correctly aligned, with no focusing offset and tilt angle between the incident and output modes. The two waists (incoming beam and output fiber) will also be supposed to be equal, as the fibers are located in the same array. Under these assumptions, crosstalk evaluation will depend only on the distance $\Delta r$ between a given incident input mode replica position $P_{l m n}^{k}$ [corresponding to a previously calculated $k$ grating, as given by (3)] and the present tested output fiber position $\left(a_{j+1}, b_{j+1}\right)$. The intensity coupling ratio between the incident mode and the fiber will be given by

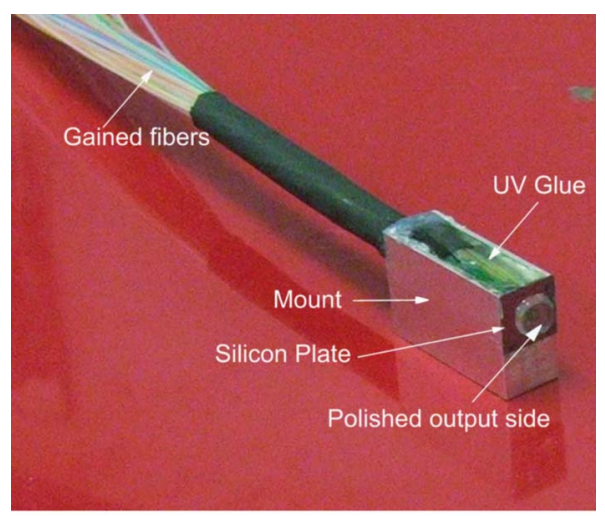

(a)

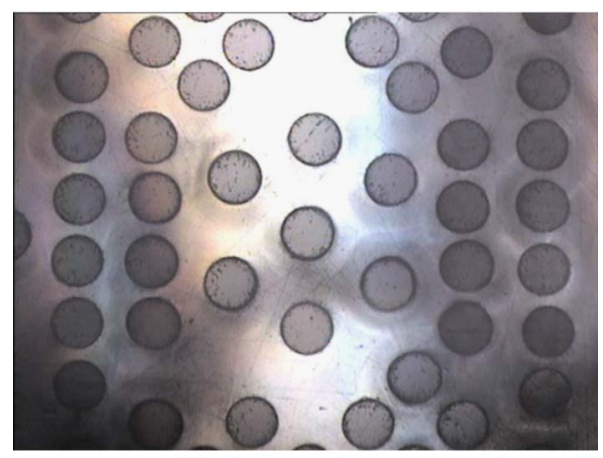

(b)

Fig. 8 (a) Fiber-array packaging view. (b) Exit side of the fiber array after the polishing stage (64 fibers have been threaded).

$I_{\text {coupl }}(\Delta r)=\left|C_{n l m}^{k}\right|^{2} \exp \left(-\frac{\Delta r^{2}}{\omega_{0}^{2}}\right)$,

where $\omega_{0}$ is the waist radius of both the incident Gaussian beam and the output fiber. The resulting power isolation value (normalized with respect to the diffraction efficiency) will be given by the relation

$$
\begin{aligned}
X(d B) & =10 \log _{10}\left(\frac{I_{\text {coupl }}}{\left|C_{100}^{k}\right|}\right) \\
& \approx-4.3\left(\frac{\Delta r}{\omega_{0}}\right)^{2}+20 \log _{10}\left(\frac{\left|C_{n l m}^{k}\right|}{\left|c_{100}^{k}\right|}\right) .
\end{aligned}
$$

In order to process the crosstalk issue in an efficient manner in the fiber-positioning algorithm, one approach is to define forbidden crosstalk areas surrounding higher-orders positions so that future fibers cannot be placed if the port isolation is worse than a given reference value $X_{0}$.

As an example, we consider the higher diffraction order shown in Fig. 4 and located at position $P_{l m n}^{k}$. Using Eq. (6), a radius $\Delta r\left(X_{0}\right)$ is calculated so that an isolation value better than $X_{0}$ is obtained from (6) if the output fiber is placed outside a disk of center $P_{l m n}^{k}$ and radius $\Delta r\left(X_{0}\right)$. An additional safety radius margin corresponding to a fiber diameter $d$ is added to compensate for possible fiber placement errors occurring during the fabrication process. This finally means that no optical fiber core center will be allowed in a disk of center $P_{l m n}^{k}$ and radius $R=\Delta r\left(X_{0}\right)+d$. 


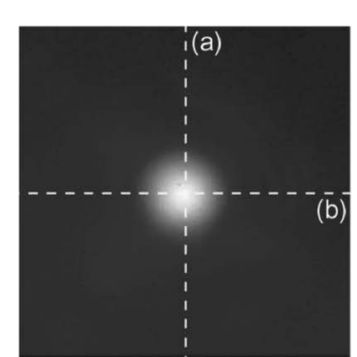

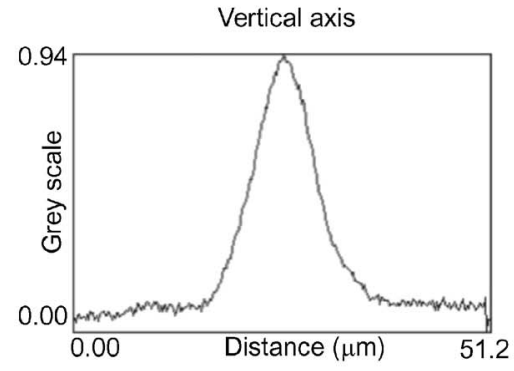

(a)

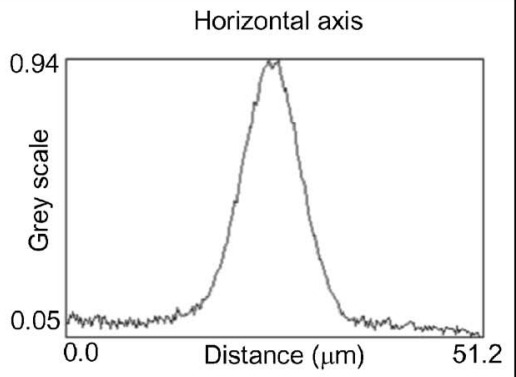

(b)

Fig. 9 Result of polished output fiber beam shape with Gaussian profile measured with near-field experimental setup.

\subsection{Fibre Array Design Algorithm}

\section{Principle}

Considering the beam-steering model described so far, the next step will consist of designing a fiber-array topology meeting the two major constraints pursued for the holographic switch i.e., (1) high-capacity and (2) low crosstalk levels. We propose an iterative procedure depicted in Fig. 5 , for which the basic idea consists of sweeping the grating period space. For each $\left(N_{x}, N_{y}\right)$ value, the placement of a fiber at the addressed position (i.e., $[1,0,0]$ th, deflected output peak location) will be validated or not, depending on the following three conditions:

1. Diffraction efficiency constraint: The first step of the fiber placement process is to select an $\left(N_{x}, N_{y}\right)$ pair greater than a specified value $\left(N_{x}, y>4\right)$ corresponding to high steering efficiency in the first diffracted order (see Fig. 3).

2. Minimal distance between fibers: The fiber placement density is limited by the minimum spacing $\delta$ between two adjacent fibers. Considering a standard SMF fibers with a $125-\mu \mathrm{m}$ cladding diameter, we will choose $\delta=130 \mu \mathrm{m}$. Using thinned cladding optical fibers (down to $40 \mu \mathrm{m}$ ) is technically feasible but will not be considered here.

3. Crosstalk condition: The position and intensity of all the diffraction orders in the output plane are calculated using (3). The power isolation of each $k$ input mode replica in the present $\left(N_{x}, N_{y}\right)$ output fiber is calculated and compared to a reference threshold $\left(X_{0}=40 \mathrm{~dB}\right.$ is the standard value). Equation (6) of the crosstalk forbidden zone is derived and transformed into an equation in $N_{x}$ and $N_{y}$ to determine the grating period that will be rejected.

At last, if all criteria are respected, the new position corresponding to the $\left(N_{x}, N_{y}\right)$ pair is accepted. If not, the position is rejected.

\subsubsection{Capacity estimation}

The fiber-addressing capacity intuitively depends on both the fiber core diameter $d$ and the size of the addressed space in the output plane per dimension, given by $\Omega=\lambda f / p$ ( $p$ is the pixel pitch). The inversely proportional relationship between the number of pixels per period $N_{x}$ and the output position $a$ reached by the first order $\left(a=\sigma / N_{x}\right)$ generates densely populated areas at the center of the addressing zone (for large $N_{x}$ values) and sparsely populated areas away from the center (smaller $N_{x}$ values). Thus, an estimate $N$ of the holographic switch capacity per dimension can be given $^{11}$ by

$N=2\left(\sqrt{\frac{\Omega}{d}}-1\right)$.

For the standard parameter values as used in Ref. 7 ( $p$ $=13 \mu \mathrm{m}, f=40.5 \mathrm{~mm}, d=125 \mu \mathrm{m}$, and $\lambda=1.55 \mu \mathrm{m})$, one obtains a $2 \mathrm{D}$ capacity estimate of $N^{2}=110$ fibers. It is expected, however, that this value will be exceeded by the results obtained with the truly $2 D$ geometrical search performed by the iterative algorithm described above.

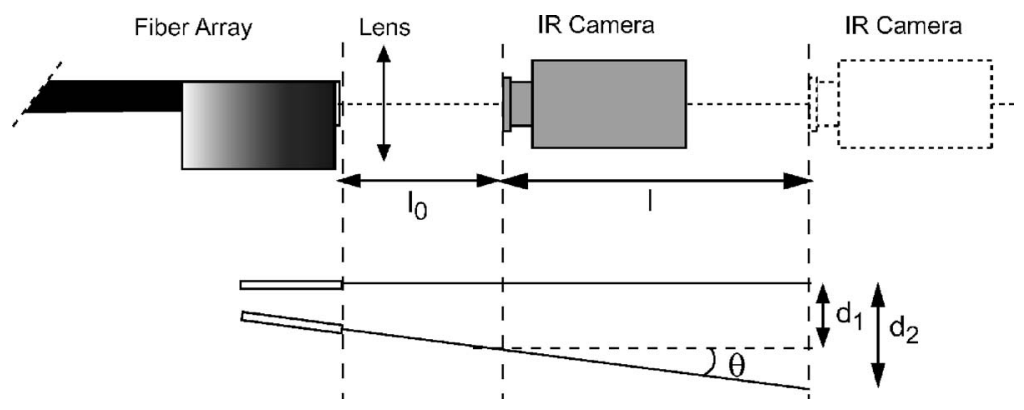

Fig. 10 Output beam angular test setup. 


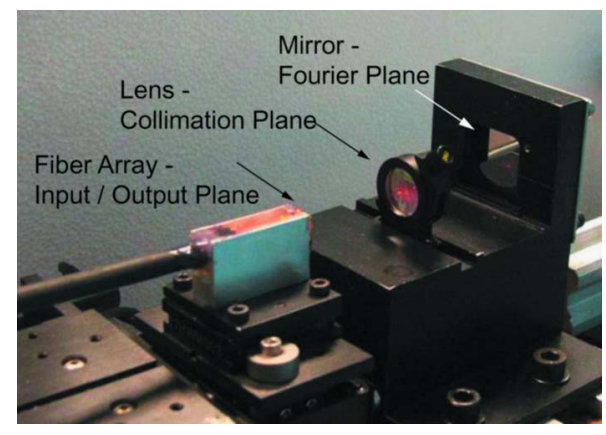

Fig. 11 Fiber-to-fiber coupling loss measurement setup.

\subsection{Results}

The algorithm shown in Fig. 5 is implemented using a computer-controlled procedure. The algorithm parameter values are intrinsically linked to such experimental conditions as SLM physical properties, optical setup dimensions, and fiber array technology. Thus, the fiber placement process will generate a non-unique solution, depicted in Fig. 6. The base pattern (A-zone) contains 32 fibers configured on an irregular lattice. It is replicated by a vertical axial symmetry to form the D-zone. Point symmetries with respect to the central fiber (denoted by "E") provide the C- and B-zone, respectively. Nine other fibers are placed along the $x$ - and $y$-axes of the output plane. This finally gives a total capacity of 141 fibers located in a $1.2-\mathrm{mm} \times 1.2-\mathrm{mm}$ square, this topology meets the previously defined crosstalk requirements. This configuration permits us to exploit the symmetrical position of diffraction orders generated by binary phase gratings. This property can be useful to implement a $1+1$ bridging function in the switching device, ${ }^{7}$ providing natural optical protection of the WDM network layer.

The layout shown in Fig. 6 will be used as a reference pattern to fabricate 2D matrices, from low capacities $(<10$ ports) to several tens of output fibers.

\section{Fiber-Array Fabrication}

\subsection{D Fiber Holder Etching}

The first step in the fiber-array manufacturing process is the choice of a 2D fiber holding technology. In this context, a well-known technology consists of superimposing 1D V-grooves, ${ }^{12}$ which enables the fabrication of fiber arrays in various materials with a high positioning accuracy. As an alternative solution, a fiber-array fabrication process in which the fibers are embedded in adjacent ceramic ferrules arranged in a honeycomb topology has been proposed. ${ }^{13}$ The device was equipped with individual micro-optics at the end face of the fibers in order to enlarge the mode diameter and thus release the alignment constraints related to optical free space interconnections. A third technology option is based on micro-holes etching through a silicon plate. This mechanical holding system is used to minimize the fiber location and pointing errors, provided that the hole depth is long enough to guide and maintain the fiber in the reference position. A reasonable value for the drilled holder thickness is four times the fiber cladding diameter, i.e., about $500 \mu \mathrm{m}$ for standard SMF fibers. This method also

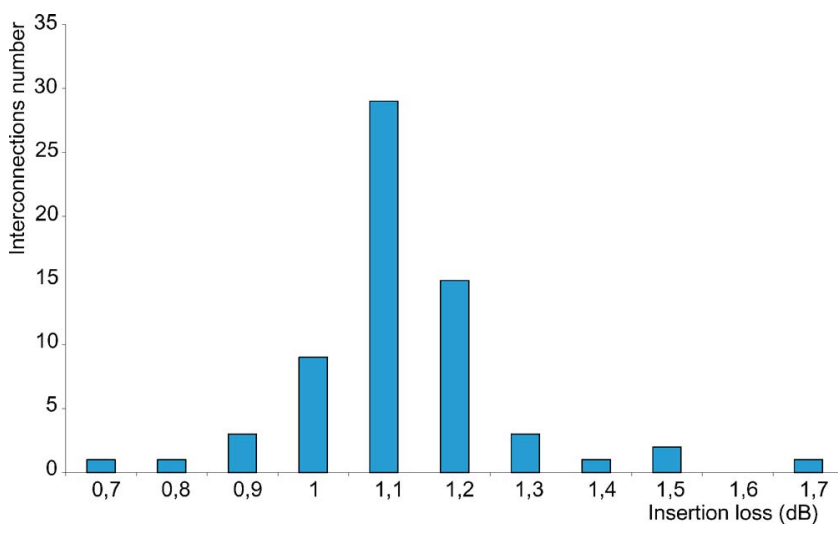

Fig. 12 Fiber-to-fiber coupling loss results for a 32-output fiber array.

provides high flexibility and accuracy in the fiber placement scheme. Due to the fiber density required (as shown in Fig. 6), this is the preferred option here. The basis for our fiber-array integration process is a silicon plate holder (530- $\mu \mathrm{m}$ thickness), whose thermal expansion coefficient is known to be very low, containing precisely etched holes placed as in Fig. 6. This micro-drilled plate is fabricated using a complex deep reactive ion etching (DRIE) process, providing holes with conical shapes on one side for an easier fiber insertion. The measured entrance and exit hole diameter specifications are $130 \pm 2.0 \mu \mathrm{m}$ and $126 \pm 1.0 \mu \mathrm{m}$, respectively. Although controlling the etched holes profiles with such an accuracy is a complex task, several batches of components were successfully etched and cleaned [Fig. 7(a)]. The fibers are then precisely inserted in the desired etched holes using a threading process controlled with a binocular microscope. The fibers are then fixed to the silicon plate with an Epotecny NOA 61 UV glue dedicated to the integration of opto-mechanical systems. This operation is achieved in a vacuum chamber in order to fill the gap between the fiber claddings and the hole walls. Each fiber is then reinforced with a polymer sheath whose diameter $d_{s}$ depends on the final fiber-array capacity (typically, $d_{s}=900 \mu \mathrm{m}$ for capacity $\leq 32$ fibers and $d_{s}=600 \mu \mathrm{m}$ for capacity $\geq 32$ fibers).

Four types of fiber arrays with different capacities of 16 , 32, 64, or 128 fibers are manufactured using the fiber holder shown in Fig. 7(a).

\subsection{Fiber-Matrix Packaging}

In a second step, a steel mount is specifically machined to the silicon plate geometry, which is precisely fixed to the output face of the steel mount using Epotecny UVS-91 UV glue. This operation will determine the final ouput fiberplane direction. As illustrated in Fig. 8(a) the optical fibers are then collected with a heat-retractable sheath that is interlocked with the steel mount using liquid NOA $61 \mathrm{UV}$ glue, so that the optical fiber,array remains mechanically reinforced during the next critical polishing step.

The polishing phase is performed with a custom procedure to maintain flatness and optical quality across the array. The fiber-to-fiber coupling ratio will directly depend upon the quality of the polishing step. The difficulty here is to impose the right fiber end-face orientations (i.e., right 
angle with respect to the fiber axes) by polishing all the fibers simultaneously and in the same conditions. To this aim, a custom-designed stainless steel polishing clamp is used to fix the fiber-array mount during the whole polishing operation. The fiber array is polished using a horizontal rotating platen with an abrasive surface whose granularity is gradually reduced from $50 \mu \mathrm{m}$ to $0.3 \mu \mathrm{m}$. The material residue draining process is optimized in order to avoid weakening of the output fiber surface. This point is periodically controlled during the process by observing the output surface through a binocular microscope. Figure 8(b) shows a typical image of an acceptable end-face quality for the fiber array after the polishing stage (64 fibers have been threaded).

\section{Experimental Test Results}

The purpose of the fiber-array characterization procedure is twofold. First, we will evaluate the optical quality of the fiber-array end face in order to validate the device fabrication process; second, the fiber matrix is tested in an optical fiber-to-fiber interconnection setup to evaluate the fiber-tofiber insertion loss.

\subsection{Output Fiber-Plane Test}

\subsubsection{Near-field tests}

The near-field experimental setup is used to measure the output fiber-beam shape. An infrared camera ${ }^{*}$ is placed on the fiber-array optical axis, and optical beam profile measurement software is used to display the optical beam profile in both the vertical and horizontal axes. Figure 9 shows the measured intensity profile in two figure orthogonal directions, featuring the ideal case of a single-mode Gaussian beam emerging from a fiber end face with good optical quality.

\subsubsection{Output beam angular tests}

The same experimental setup principle is used to test the output fiber-surface orientation. The infrared camera is placed here at two different positions of the optical axis in order to compare the positions of the Gaussian beam center between a reference fiber and the tested fiber (cf. Fig. 10). The angular deviation $\delta \theta$ of the beam emerging from the tested fiber is determined by the equation

$\tan (\delta \theta)=\frac{\left(d_{2}-d_{1}\right)}{l}$,

where $d_{1}$ and $d_{2}$ are the two distances between the incident Gaussian beam centers from the two fibers measured at two positions separated by a distance $l$. The angular deviation can then be used to estimate the coupling efficiency variation between the input and ouput fibers (the latter is supposed to be perfectly aligned on-axis):

\footnotetext{
*Type IR-Vidicon camera; Model C2400-03; sensitive surface 12.9(H)9.5(V); horizontal lines: 575 (CCIR).
}

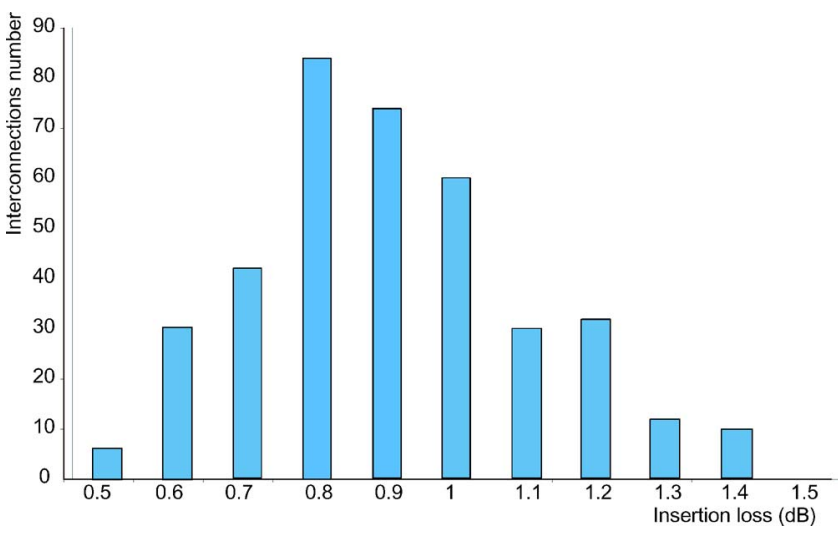

Fig. 13 Fiber-to-fiber coupling loss results for a 64-output fiber array.

$I_{\text {coupled }}(\delta \theta)=\exp \left[-\left(\frac{\pi \omega_{0}}{\lambda}\right)^{2} \cdot \delta \theta^{2}\right]$.

The angular tests performed on a statistical set of 20 fibers have shown a negligible angular deviation, as they produce an average coupling efficiency variation lower than $0.1 \mathrm{~dB}$.

\subsection{Fiber-to-Fiber Coupling Loss Measurement}

An important step to assess the performance of the fiber matrix is to test the fiber-to-fiber coupling loss. This is achieved by replacing the SLM of Fig. 1 with a mirror optimized for near-infrared operation. The lens used is an achromat doublet (Melles-Griot) composed of two different glasses $\left(\mathrm{BaLKN} 3\right.$ and $\left.\mathrm{SF} 15^{\dagger}\right)$. The fiber array is placed on 6 -axis micro-positioning system in the reflective $4 f$ setup shown in Fig. 11.

The optical micro-bench is first aligned using a visible $\mathrm{He}-\mathrm{Ne}$ laser light that is injected in the input fiber and coupled to an output fiber by moving the fiber array. The fiber-to-fiber coupling loss is then measured using infrared light at $1.55-\mu \mathrm{m}$ wavelength. The operation is repeated for a large number of input/output fiber pairs. Test results are proposed in Figs. 12 and 13 for 32 and 64 output fiber arrays, respectively, with a collection of more than 100 measurements between randomly chosen fiber pairs. The mean measured fiber-to-fiber losses are, respectively, $1.1 \mathrm{~dB}$ and $0.9 \mathrm{~dB}$, with $0.3-\mathrm{dB}$ standard deviation in both cases. These values are encouraging as the fiber end faces are not antireflection-coated.

The final test consists of measuring the polarizationdependent coupling loss variation. It is performed on a set of randomly chosen fibers (the fiber array used is the component containing 64 fibers) by inserting a polarization controller between the emitted fiber and the addressed fiber. The fiber-to-fiber coupling efficiency variations between the maximum and minimum values for different polarization states are given in Table 1 and reveal a negligible polarization-dependent loss for the tested fiber array.

\footnotetext{
Model \#01LAL011.
} 
Table 1 Polarization-dependent loss measurement (64-fiber array).

\begin{tabular}{lcc}
\hline \hline Fiber $F_{i}$ & $\operatorname{PDL} \lambda / 4$ & $\operatorname{PDL} \lambda / 4+\lambda / 2$ \\
\hline$F_{1}$ & 0.027 & 0.0662 \\
$F_{2}$ & 0.030 & 0.0670 \\
$F_{3}$ & 0.049 & 0.0850 \\
$F_{4}$ & 0.036 & 0.0760 \\
$F_{5}$ & 0.032 & 0.0700 \\
Referential measure & 0.020 & 0.0350 \\
\hline
\end{tabular}

\section{Conclusions}

We have reported the design of a 2D irregular fiber array dedicated to fiber-to-fiber holographic switching using a binary-phase steering device. We have proposed a specific high-density output fiber placement algorithm based on coupling efficiency maximization and crosstalk minimization.

Fiber-array prototype fabrication steps have been described, and two devices have been tested. The best component exhibits a 64-output fiber capacity with less than $1 \mathrm{~dB}$ fiber-to-fiber insertion loss and negligible polarization-dependent loss.

The next step is to integrate the fabricated fiber array in a dynamic holographical fiber-to-fiber switching setup. This operation as well as the performance of the resulting device are presented in a submitted publication. ${ }^{14}$

\section{Acknowledgments}

The authors would like to thank the Optogone/Memscap and PHS MEMS companies for funding and performing the wafer-etching process, and Michel Gadonna (ENST Bretagne) for valuable discussions concerning the nearfield characterization setup.

\section{References}

1. J. Strand and A. Chiu, "Realizing the advantages of optical reconfigurability and restoration with integrated optical cross-connects," $J$. Lightwave Technol. 21(11), 2871-2882 (2003).

2. P. A. Bonenfant and M. L. Jones, "OFC 2003 workshop on wavelength selective switching based optical networks," J. Lightwave Technol. 22, 305-309 (2004).

3. T. Goh, M. Ishii, T. Mizuno, S. Kamei, I. Ogawa, H. Hirota, Y Tamura, M. Kobayashi, M. Yanagisawa, S. Sohma, and A. Kaneko, "Four-degree hub switch module using multi-chip planar lightwave circuit integration technology for transparent roadm ring interconnection," in Optical Fiber Communication Conference Technical Digest, pp. OTuF3.1-OTuF3.3 (2006).

4. D. M. Marom, D. T. Neilson, D. S. Greywall, C.-S. Pai, N. R. Basavanhally, V. A. Aksyuk, D. O. López, F. Pardo, M. E. Simon, Y. Low, P. Kolodner, and C. A. Bolle, "Wavelength-selective $1 \times \mathrm{k}$ switches using free-space optics and mems micromirrors: Theory, design, and implementation," J. Lightwave Technol. 23(4), 1620-1630 (2005).
5. G. Baxter, S. Frisken, D. Abakoumov, H. Zhou, I. Clarke, A. Bartos, and S. Poole, "Highly programmable wavelength selective switch based on liquid crystal on silicon switching elements," in Optical Fiber Communication Conference Technical Digest, pp. OTuF2.1OTuF2.3 (2006).

6. W. Crossland, I. G. Manolis, M. M. Redmond, K. L. Tan, T. D. Wilkinson, M. J. Holmes, T. R.Parker, H. H. Chu, J. Croucher, V. A Handerek, S. T. Warr, B. Robertson, I. G. Bonas, R. Franklin, C. Stace, H. J. White, R. A. Woolley, and G. Henshall, "Holographic optical switching: The "roses' demonstrator," J. Lightwave Technol. 18(1), 1845-1854 (2000).

7. B. Fracasso, J. L. de Bougrenet de la Tocnaye, M. Razzak, and C. Uche, "Design and performance of a versatile holographic liquidcrystal wavelength-selective optical switch," J. Lightwave Technol. 21(10), 2405-2411 (2003).

8. K. Heggarty, B. Fracasso, C. Letort, J.-L. de Bougrenet de la Tocnaye, M. Birch, and D. Krüerke, "Silicon backplane ferroelectric liquid crystal spatial light modulator for uses within an optical telecommunications environment," Ferroelectrics 312, 39-55 (2004).

9. K. Tan, S. T. Warr, I. G. Manolis, T. D. Wilkinson, M. M. Redmond, W. A. Crossland, R. J. Mears, and B. Robertson, "Dynamic holography for optical interconnections. II Routing holograms with predictable location and intensity of each diffraction order," J. Opt. Soc. Am. A 18(1), 205-214 (2001).

10. C. Letort, "Modélisation, implantation el caractérisation de fonctions de routage tri-dimensionnel de canaux optiques pour les réseaux WDM," in PhD dissertation, Université de Rennes 1, pp. 149-154 (2007).

11. C. Letort, M. Razzak, B. Fracasso, and K. Heggarty, "Dynamic spectral response of a liquid crystal based optical channel selector," in PS 2003-Photonics in Switching Conference, pp. 259-261 (2003).

12. Corning, http://www.corning.com/docs/specialtymaterials/pisheets/ pi107.pdf.

13. P. Chanclou, M. Thual, J. Lostec, P. Auvray, J. Caulet, G. Joulie, A Poudoulec, and B. Clavel, "Highly efficient collective coupling between laser diode arrays," Proc. SPIE 3289, 97-102 (1998).

14. C. Letort, B. Fracasso, and K. Heggarty, "Design and test of a 1 $\times 64$ and crosstalk-free 2D-holographic liquid-crystal optical switch," J. Lightwave Technol. submitted for publication.

Cedric Letort was a PhD student from GET/Ecole Nationale Supérieure des Télécommunications de Bretagne (Brest, France). He received an engineering degree from Magistère Matériaux de Rennes I (France) and a PhD degree in Signal Treatment \& Telecommunications from Université de Rennes I and Ecole Nationale Supérieure des Télécommunications de Bretagne in 2007. He is currently involved in engineering projects on optical instrumentation for satellite application at CNES (Toulouse, France).

Bruno Vinouze has been an associate professor at TELECOM Bretagne in the Optical Department since 2001. He graduated from INSA in Physics in 1996. In a France Telecom Research Center he has over 20 years of experience in the liquid crystal domain in LC displays (2S and $3 S$ TFTLCD) and specific telecom LC devices (switches, VOA, MUX, tunable filters). Between 1999 and 2001 he has been involved in R\&D for different telecom start-up. He lectures in the telecom physic layers, especially on optical components. He is the co-author of more than 46 publications and has 26 patents.

Bruno Fracasso is an associate professor at GET/Ecole Nationale Superieure des Telecommunications de Bretagne (Brest, France). $\mathrm{He}$ received an engineering degree from Ecole Nationale Supérieure des Telecommunications de Bretagne in 1989 and a $\mathrm{PhD}$ degree in Electrical Engineering from Université de Haute Alsave, Mulhouse (France) in 1993. He is currently involved in research projects on optical switching for and routing within fiber networks, combining device modelling, simulation and technological integration. 